\title{
Etnoarqueología de las casas Huapula y Jíbaro
}

Ethnoarchéologie des maisons Huapula et Jivaros

Ethnoarchaeology of Huapula and Jivaro houses

\section{Stéphen Rostain}

\section{OpenEdition}

Journals

Edición electrónica

URL: http://journals.openedition.org/bifea/3890

DOI: $10.4000 /$ bifea.3890

ISSN: 2076-5827

\section{Editor}

Institut Français d'Études Andines

Edición impresa

Fecha de publicación: 1 diciembre 2006

Paginación: 337-346

ISSN: 0303-7495

Referencia electrónica

Stéphen Rostain, «Etnoarqueología de las casas Huapula y Jíbaro », Bulletin de l'Institut français d'études andines [En línea], 35 (3) | 2006, Publicado el 01 junio 2007, consultado el 01 diciembre 2020. URL : http://journals.openedition.org/bifea/3890 ; DOI : https://doi.org/10.4000/bifea.3890

Les contenus du Bulletin de l'Institut français d'études andines sont mis à disposition selon les termes de la licence Creative Commons Attribution - Pas d'Utilisation Commerciale - Pas de Modification 4.0 International. 


\title{
Etnoarqueología de las casas Huapula y Jíbaro
}

\author{
Stéphen Rostain*
}

\begin{abstract}
Resumen
El valle del Upano, en la alta Amazonía ecuatoriana, está caracterizado por la presencia de numerosos montículos artificiales de tierra precolombinos. Edificados y luego abandonados por las sociedades Upano entre 700 a.C. y 400 d.C., algunos de ellos fueron reocupados por grupos Huapula entre 800 y 1200 d.C. La excavación horizontal de un montículo reveló un piso habitacional muy bien conservado, autorizando un análisis espacial de las actividades practicadas. El estudio etnoarqueológico de esta habitación Huapula muestra las grandes similitudes que existen con el modo de hábitat actual de los grupos jíbaros de la región.
\end{abstract}

Palabras clave: Amazonía, Upano, Huapula, Jíbaro, etnoarquología, hábitat

\section{Ethnoarchéologie des maisons Huapula et Jivaros}

\section{Résumé}

La vallée de I'Upano, en haute Amazonie équatorienne, se caractérise par la présence de nombreux monticules artificiels de terre précolombiens. Edifiés puis abandonnés par les sociétés Upano entre 700 av. J.-C. et 400 apr. J.-C., certains d'entre eux furent réoccupés par des groupes Huapula entre 800 et 1200 apr. J.-C. La fouille horizontale d'un tertre a mis au jour un sol domestique très bien conservé, permettant une analyse spatiale des activités pratiquées. L'étude ethnoarchéologique de la maison Huapula a montré les grandes similitudes qui existaient avec le mode d’habitat actuel des groupes Jivaros de la région.

Mot clés: Amazonie, Upano, Huapula, Jivaros, ethnoarchéologie, habitat 


\title{
Ethnoarchaeology of Huapula and Jivaro houses
}

\begin{abstract}
The Upano valley, in upper Ecuadorian Amazonia, is characterized by numerous precolumbian artificial earthmounds. Built then abandoned by Upano societies between 700 BC and 400 AD, some of them were re-occupied by Huapula groups between 800 and 1200 AD. Open-area excavations of one mound produced a well preserved domestic level, permitting a spatial analysis of activities. Ethnoarchaeological study of the Huapula house has shown great similarities with settlements of modern Jivaros groups of the area.
\end{abstract}

Key words: Amazonia, Upano, Huapula, Jivaros, ethnoarchaeology, settlement

\section{INTRODUCCIÓN}

En 1999, cuando terminaba el artículo sobre las excavaciones de montículos del valle del Upano, publiqué en esta revista interrogándome si «los Huapula se relacionaban culturalmente con los Jíbaros» (Rostain, 1999b: 85). Hoy en día es posible aportar algunos elementos para responder a esta pregunta.

La excavación por decapado del nivel cultural Huapula de un montículo artificial de tierra en el valle del Upano, reveló un piso habitacional muy bien conservado (Rostain, 1999a). El análisis espacial de los rasgos y vestigios permitió encontrar la práctica de diversas actividades y su distribución en el área de la casa (Rostain, 1999c; 2000). El estudio etnoarqueológico de esta habitación Huapula muestra las grandes similitudes que existen con el modo de hábitat actual de los grupos jíbaros.

\section{EL VALLE DEL UPANO}

Encerrado entre 2 cordilleras al pie de los Andes, el valle del Upano forma una entidad geográfica en la Alta Amazonía. El Upano nace en los Andes a 4000 m de altura, corre hacia el este hasta el pie de monte a alrededor de $1200 \mathrm{~m}$ de altura. Dibuja entonces, una brusca curva para ir hacia el sur, siguiendo un corredor delimitado al oeste por los Andes y al este por la cordillera del Cutucú. Su lecho, de 1 km de ancho, está enmarcado por abruptos barrancos de 70-100 m de altura en donde se encuentran numerosos sitios arqueológicos. El Upano está ubicado en la falla que delimita el Sub-Andino, la misma que provoca frecuentes movimientos sísmicos. El volcán Sangay se encuentra al noroeste del sitio Huapula, con una altura de $5230 \mathrm{~m}$ y un diámetro en su base de 10-12 km. Su actividad volcánica es permanente. A inicios del siglo XX, el estruendo de sus explosiones se escuchó a más de $600 \mathrm{~km}$ de distancia. La vida de los habitantes del valle del Upano ha estado desde siempre muy ligada a los caprichos del Sangay.

A pesar de esto, el valle ha sido ocupado por el hombre desde hace tiempo. Hace más de dos milenios, ciertos grupos humanos comenzaron a edificar montículos de tierra a lo largo del barranco que domina el Upano. Huapula es el sitio más extenso del alto Upano con $700000 \mathrm{~m}^{2}$ (fig. 1A). Está compuesto por unos treinta complejos de montículos y una red de largos canales. El Complejo XI bordea el río Huapula y se extiende sobre un área de 70 × $50 \mathrm{~m}$ (fig. 1A). Está organizado según el modelo espacial característico de los sitios del Upano. Es decir 6 elevaciones que rodean 2 plazas bajas, separadas por una plataforma central. Situado en una pendiente, el montículo Tola Central se eleva a 3,5 m de altura, extendiéndose su cima a alrededor de $130 \mathrm{~m}^{2}(16$ × $8 \mathrm{~m})$. La Tola Central y la plaza norte fueron excavadas por decapado de grandes superficies (fig. 1B). 

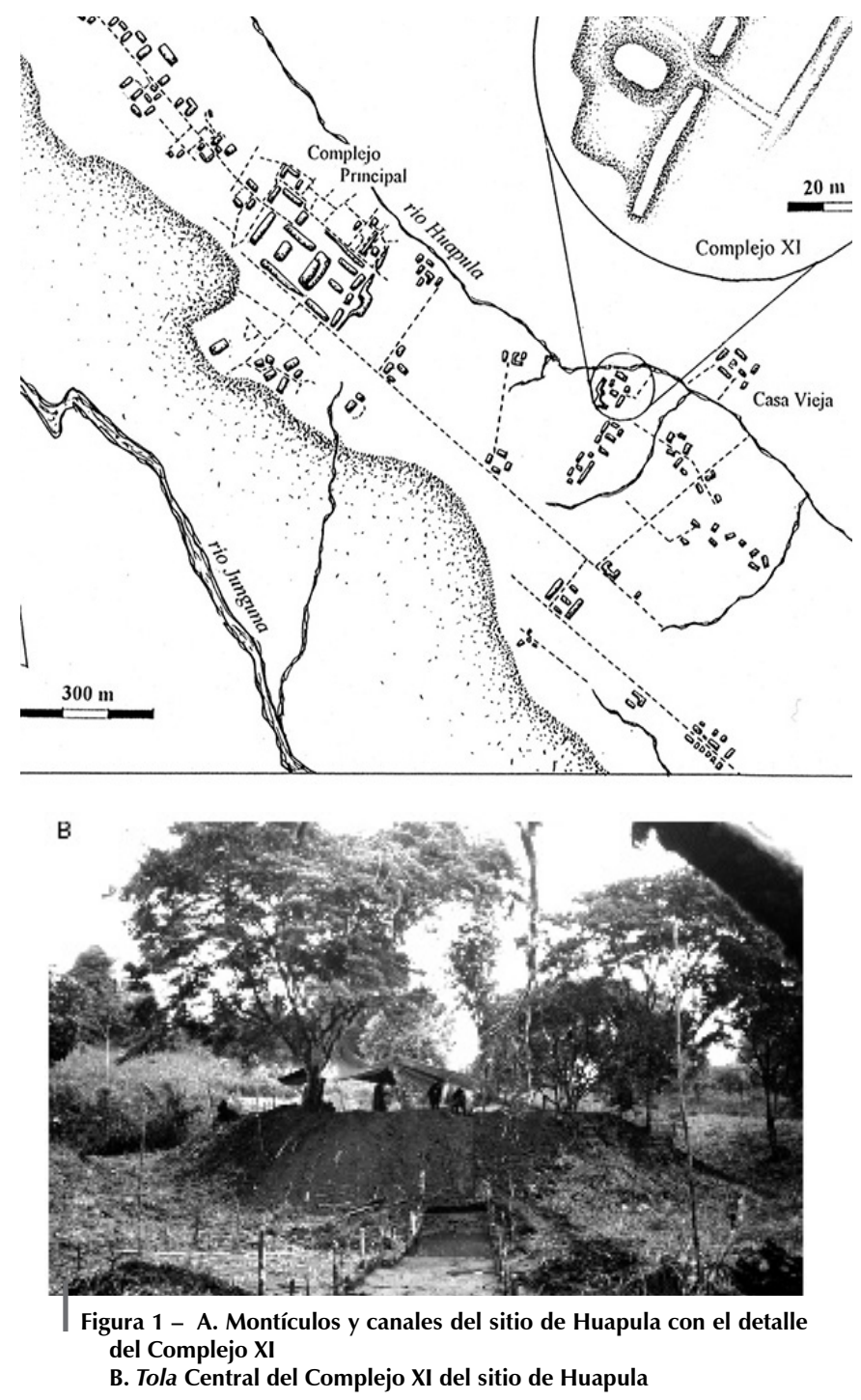

El Complejo XI domina un río de fácil acceso gracias a un camino cavado, suavizando así el desnivel para evitar los deslizamientos, especialmente en el momento de aprovisionamiento de agua. Un basural lleno de tiestos de cerámica fue localizado en los linderos del complejo, al borde del barranco sobre el riachuelo.

La estratigrafía de la Tola Central representa 4 etapas sucesivas principales: entre 700 a.C. y 400 d.C., comunidades de la cultura Upano instalaron sus pueblos a orillas del Upano. Los primeros en llegar ocuparon directamente el espacio sin realizar terraplenes.

Más adelante construyeron montículos de tierra, plazas bajas, canales y caminos cavados, distribuidos en complejos con un modelo espacial preciso. La cima del montículo de arcilla de la Tola Central fue quemada para así conseguir un piso compacto. 
Hacia el 600 d.C., una erupción del Sangay depositó una gruesa capa de cenizas en el valle del Upano provocando la huida de los habitantes. Del 800 al 1200 d.C., llegaron al valle grupos de cultura Huapula y se asentaron sobre los montículos pre existentes.

Tres conjuntos cerámicos principales fueron reconocidos: el Upano es el más antiguo, típico por su decorado en rojo y delimitado por incisiones, y más adelante reemplazado por la cerámica Kilamope, caracterizada por incisiones, impresiones cordeladas y pintura negativa marrón y beige. La cerámica precolombina más reciente es la Huapula con un decorado corrugado o pintada de rojo y blanco.

\section{LA CASA HUAPULA}

En la Amazonía, el desecamiento del área de hábitat constituye una prioridad. Se cavan zanjas alrededor de las casas para favorecer el drenaje. Los altos son áreas de asentamiento privilegiadas. $\mathrm{Al}$ instalar su casa en la cima de un montículo, el grupo Huapula se protegía de las inundaciones, facilitaba la evacuación de las aguas y evitaba la formación de un suelo enlodado (fig. 1B). Al igual que en la casa precolombina de la Tola Central, en la actualidad los achuar construyen en los altos de un riachuelo más que al borde del mismo, e intentan orientar la casa de forma paralela a éste (Descola, 1986: 154).

En el Complejo XI, dado que la pendiente que llevaba al río era demasiado abrupta, fue necesario atenuarla cavando un camino menos empinado. Igualmente, los actuales achuar aseguran el acceso al río instalando leños en forma de escalera para así prevenir los deslizamientos peligrosos (Descola, 1986: 139).

Las excavaciones arqueológicas antes realizadas en la Amazonía, consistían en pequeños sondeos estratigráficos. Durante el proyecto Sangay-Upano (1995-1998), se adoptó una nueva técnica de excavación que decapó grandes superficies y dio una visión global del piso arqueológico. El análisis de la dispersión de los artefactos y rasgos culturales ayuda a la reconstrucción de la utilización del espacio doméstico.

El decapado de $90 \mathrm{~m}^{2}$ en la cima de la Tola Central sacó a la luz del día los restos de un piso doméstico y las huellas de 49 hoyos de poste. En nuestros días, estos últimos se hacen con un palo de chonta afilado y se extrae la tierra con la mano. Cuando ésta ya no basta, se utiliza un palo de guadúa, partido en la punta, de manera a que se llene de tierra con la presión (Bianchi \& AAVV, 1982).

El estudio planimétrico de los rasgos permite esbozar la forma de una estructura que cubre casi la totalidad de la superficie utilizable, alrededor de $130 \mathrm{~m}^{2}$. La superficie máxima de la casa Huapula es de unos $80 \mathrm{~m}^{2}$. Esto quiere decir que una casa ocupaba la cima del montículo y sus dimensiones eran comparables a la casa Shuar o Achuar actual. Cinco dataciones al ${ }^{14} \mathrm{C}$ han sido obtenidas sobre muestras de carbones de madera fiables que provenían del piso habitacional:

$\begin{array}{lll}\text { BETA-100537 } & 1210 \pm 80 \mathrm{BP} & 692-892 \mathrm{DC} 1 \\ \text { BETA-100538 } & 1070 \pm 70 \mathrm{BP} & 892-1023 \mathrm{DC} \\ \text { BETA-100308 } & 940 \pm 60 \mathrm{BP} & 1031-1155 \mathrm{DC} \\ \text { BETA-106087 } & 850 \pm 60 \mathrm{BP} & 1055-1259 \mathrm{DC} \\ \text { BETA-100539 } & 770 \pm 60 \mathrm{BP} & 1211-1285 \mathrm{DC}\end{array}$

Está claro que la casa no estuvo ocupada durante 500 años, sino únicamente durante un periodo que se sitúa entre las fechas propuestas.

\footnotetext{
${ }^{1}$ Calibración 1 Sigma. Calib Radiocarborn Calibration Program rev.4.3 (c) 1986-2005 M. Stuiver \& P.J. Reimer.
} 


\section{ACTIVIDADES DOMÉSTICAS}

En la casa Huapula existían 4 fosas redondas u ovaladas de 40 a $80 \mathrm{~cm}$ de diámetro cada una.Dos fosas se encontraban vacías y una tercera ubicada en el centro contenía una gran olla de cerámica (figs. 2C, D). Inestables debido a su base redonda, las ollas debían ser aseguradas para no voltearse. Al igual que en las casas amerindias, podían estar parcialmente enterradas en una fosa. La última fosa localizada hacia el borde oriental del montículo, era profunda y cubierta por la parte superior de una gran olla (fig. 2A), que sirvió aparentemente para tapar la fosa que pudo contener alimentos (fig. 2B), esto dado a que no se halló ningún tiesto del fondo del recipiente.

Los 7 fogones simples descubiertos tenían un diámetro de 25 a $45 \mathrm{~cm}$ (fig. 3B). Se trataba de estructuras de combustión instaladas al nivel del piso, sin adecuaciones ni excavaciones. El fogón estaba constituido por una capa de arcilla irregular de color rojo. Algunos experimentos llevados a cabo sobre el sitio demostraron que el suelo arcilloso de color amarillo enrojecía con el fuego. Luego de 1 ó 2 años, los fogones en los que se hicieron estas experimentaciones presentaron un aspecto similar a aquellos encontrados durante la excavación. Al centro, 3 de ellos formaban una gran y única área de combustión. Están en efecto conectados por un área de suelo compacto, duro y rojizo que contiene numerosos carbones de madera y granos calcinados, lo mismo que se interpreta como el resultado de la dispersión de los fogones. Fenómenos similares ocurren hoy en las casas Jíbaros:
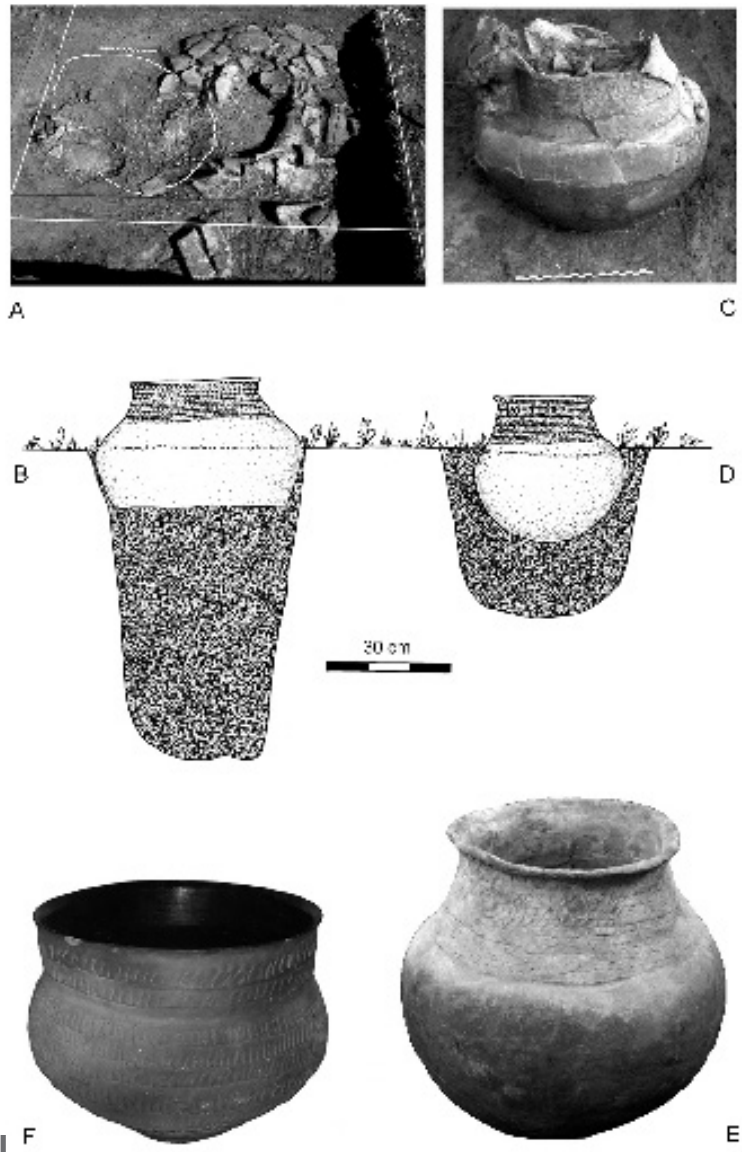

Figur

Figura 2 - A. Parte superior de la olla 4 aplastada sobre una fosa señalada por un círculo punteado blanco

B. Reconstitución de la boca de la fosa con la olla 4 cortada

C. Olla 1 hallada entera en la fosa

D. Olla 1 enterrada en fosa

E. Olla Jíbaro actual

F. Olla Huapula 1

« (...) en la vecindad inmediata de los fogones (...) abundantes cantidades de residuos materiales fueron compactadas en las acumulaciones de ceniza pisoteada, las cuales conectaban los fogones individuales. Fenómeno debido, por supuesto, a la alta frecuencia de uso en la preparación cotidiana de los alimentos, donde los desechos de los mismos son constantemente incorporados en los depósitos pisoteados de ceniza blanda alrededor de los fogones». (Zeidler, 1983: 181)

Otros dos, ubicados al norte, formaban otra área. Los dos últimos estaban dispuestos simétricamente a $4 \mathrm{~m}$ del grupo central, uno al este y el segundo al oeste.

Como en la actualidad, había varios fogones en la casa Huapula. Los dos conjuntos centrales tenían una función culinaria. Un metate cubría uno de los fogones, indicando así que el utensilio había sido desplazado y que la organización espacial de la cocina era a veces cambiada. Los 2 fogones periféricos estaban seguramente destinados a la iluminación y como fuente de calor (el sitio está a $1000 \mathrm{~m}$ de altura). 
Durante la excavación, algunos granos calcinados fueron recuperados en el centro del montículo. Los análisis arqueobotánicos permitieron identificar 18 morfo-especies representativas de 5 familias (Mimosaceae, Passifloraceae, Phytolaccaceae, Poaceae, Rosaceae) y diversos tipos de hongos. Pocos restos botánicos han sido hasta el momento hallados en excavaciones en la Amazonía. Las numerosas macro-plantas calcinadas recolectadas en la Tola Central son interesantes por su diversidad y por su presencia en un contexto doméstico y culinario. El maíz domina ampliamente en el mostrario. Entre las demás plantas de consumo, ya sean salvajes o cultivadas, se identificaron guabas, cerezas, moras y granadillas. Sin embargo, ciertas especies tienen también un uso medicinal como las Inga, Prunus y Phytolacca.

En el lado oeste del montículo, no muy lejos de uno de los fogones, se hallaban 2 torteros de cerámica, que servían para hilar el algodón, materia prima ampliamente explotada por los pueblos amazónicos. Podemos suponer que la fabricación del hilo se hacía en este espacio.

\section{COMPARACIÓN DE LAS CASAS HUAPULA Y JIVARO}

Se puede comprender mejor la distribución espacial de los artefactos y rasgos culturales de la Tola Central, gracias a un análisis etnoarqueológico de las viviendas contemporáneas de la región, como aquel llevado por Zeidler (1983) con los achuar de la provincia de MoronaSantiago. En efecto, se observan numerosas similitudes entre las casas Huapula y Achuar. El grupo Achuar (familia lingüística Jíbaro) ocupa hoy el valle del Pastaza, al este del Upano, a una altura de 200 a $500 \mathrm{~m}$.

El patrón de asentamiento Achuar se caracteriza por la casa familiar aislada que constituye la unidad de base. En ciertos casos, 2 ó 3 casas dispersas pueden ocupar una misma área. La casa Achuar tiene forma elíptica con un tejado de caballete (fig. 5A), está generalmente desprovista de paredes externas, salvo en situaciones de conflicto en donde se la cierra y se la protege eventualmente por una alta palizada. Generalmente, sus dimensiones son de 13-15 m de largo por 8-10 m de ancho y 5-7 m de alto (Bianchi \& AAVV, 1982). Son excepcionalmente grandes, así, una vivienda de 20 habitantes permanentes medía 23 por 12 m (Descola, 1986: 141). La mayor parte del tiempo, la residencia uxorilocal se compone por una casa que alberga de 5 a 20 personas, es decir al jefe de familia, sus esposas e hijos (Descola, 1986; Zeidler, 1983). La casa Achuar y Shuar está organizada según una dicotomía sexual (Harner, 1995). Si durante la noche, las parejas duermen juntas en diferentes lugares de la casa, el espacio se divide en 2 durante el día: un sector para las mujeres (ekent) y otro para los hombres (tankamash). La cocina y los recipientes se encuentran en la zona femenina. En la masculina en cambio, los bancos permiten recibir a los visitantes, tomar la chicha, comer y fabricar artefactos.

Cada adulto ocupa un espacio privilegiado para efectuar todo tipo de tareas, y cada mujer dispone de un área personal con un fogón y sus bienes materiales (Zeidler, 1983). Ciertas tareas y actividades son únicamente realizadas en lugares bien definidos. Así, el maíz es molido en un solo lugar y la chicha consumida en un espacio social central. De la misma manera, la chicha, que se consume en gran cantidad, se conserva aparte. Cada mujer conserva una o más ollas (muits) llenas de chicha junto con cuencos para servirla, en un área de almacenaje. El espacio donde se bebe la chicha es utilizado por ambos sexos. Dos actividades típicamente masculinas se realizan en la periferia de la casa: el afilado de machetes y la fabricación de cerbatanas (fig. 4A). Las camas sobre plataforma (peak) están dispuestas en el contorno de la casa, con un fogón ubicado generalmente cerca al pie de cada una.

La reconstitución de las áreas de actividad de la casa Huapula da como resultado una distribución similar a aquella de la casa Achuar (fig. 4B). La molienda del maíz y la cocina se practicaban en el centro. La presencia de 2 metates y sus manos cerca de los fogones centrales y de 2 torteros sugiere que 2 mujeres vivían en la casa. En efecto, en la actualidad cada esposa de una vivienda Achuar, posee sus propios utensilios: 

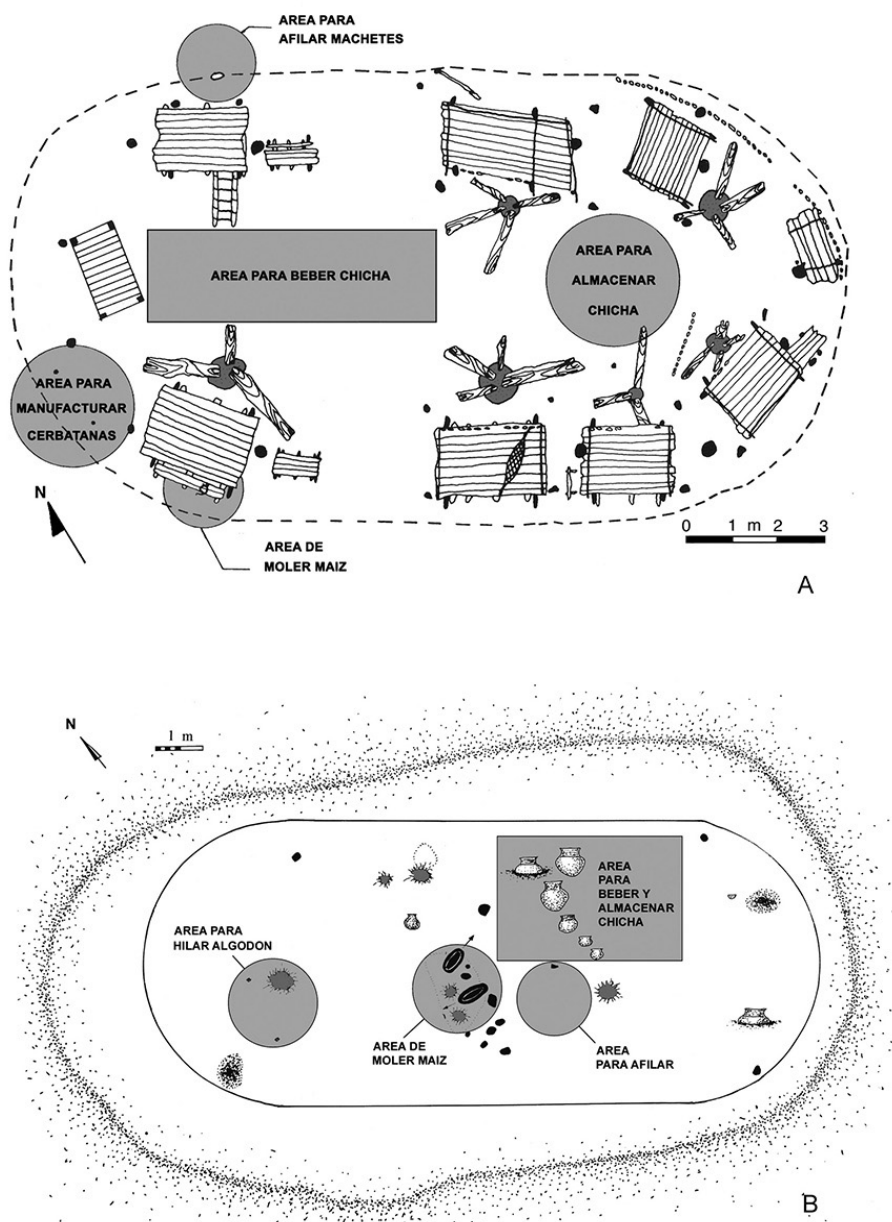

Figura 4 - A. Áreas de actividades en la casa Achuar (según Zeidler, 1983: fig. 5). Esta casa de Pumpuentza medía 18,2 x 10 m y albergaba a 16 ocupantes

B. Áreas de actividades en la casa Huapula. La excavación por decapado reveló una distribución espacial específica de ciertas tareas al igual que en la casa Jíbaro

«el conjunto de utensilios femeninos está duplicado en cada área de actividad e invariablemente está ubicado alrededor de los rasgos domésticos inmóviles, tales como el fogón central, la cama, etc.». (Zeidler, 1983: 172)

Las ollas eran colocadas justo al este de la cocina en donde la presencia de cuencos indica que la bebida era consumida en este espacio. Las ollas globulares corrugadas Huapula son muy parecidas a las ollas corrugadas Achuar actuales (figs. 2E, F), que están generalmente juntas en la zona central del sector femenino. La persistencia de una forma y de un decorado cerámico durante más de un milenio en la provincia de Morona-Santiago es un hecho notable, pero 

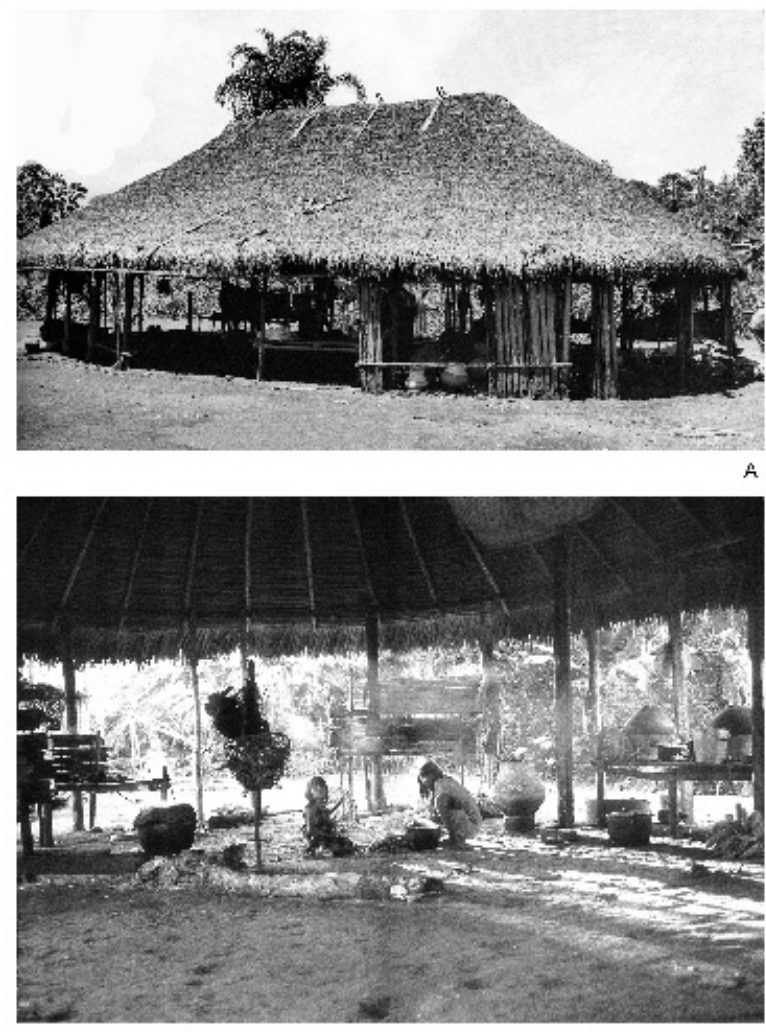

Figura 5 - A. Casa Achuar (según Trupp, 1981: 130)

B. Interior de una casa Achuar (según Descola, 1993: foto 13)

no excepcional en la Amazonía (cf. Guffroy en este volumen). El afilado se hacía cerca de la cocina. En fin, la presencia de torteros junto a un extremo, deja suponer que se hilaba el algodón en ese lugar. Los datos arqueológicos indican que la casa Huapula tenía un patrón espacial comparable al de la casa Achuar, es decir con una división del espacio doméstico en 2 sectores (masculino y femenino), así como áreas de actividades individuales y colectivas.

\section{CONCLUSIÓN}

Debido a la ausencia de datos arqueológicos de este tipo, un análisis comparativo de los diferentes hábitats amazónicos actuales fue llevado a cabo. Inclusive si existen numerosos aspectos en común, cada grupo muestra rasgos distintivos de su modo de asentamiento. La casa Huapula posee muchas características que la vuelven comparable con la casa Achuar o Shuar actual (fig. 5B). Estos pueblos ocupan por otro lado un mismo territorio.

Las diferentes características de organización interna de una casa Achuar se encuentran en las huellas de habitación dejadas por los huapula. Extendiéndose en una superficie de $80 \mathrm{~m}^{2}$, la casa Huapula presentaba un plano rectangular con los extremos rectos o redondeados. Dada su dimensión, se puede suponer que albergaba a una sola familia. Al igual que en la actualidad, el equipamiento doméstico era esencialmente utilizado por las mujeres. Los 2 metates encontrados en la cocina, así como los 2 torteros, corresponden posiblemente a la presencia de 2 mujeres que utilizaban sus propios utensilios. Esto podría ser un indicio de una sociedad polígama comparable con la de los actuales shuar. Pensamos que la cultura Huapula representa la primera aparición Jíbaro en la cuenca del río Upano, lo que indicaría una presencia de este grupo en esta región desde hace más de un milenio.

\section{Referencias citadas}

BIANCHI, C. \& AA. VV., 1982 - Artesanías y Técnicas Shuar, 477 p.; Quito: Ediciones Mundo Shuar.

DESCOLA, P., 1986 - La nature domestique. Symbolisme et praxis dans l'écologie des Achuar, 450 p.; Paris : Fondation Singer-Polignac/Editions de la Maison des Sciences de I'Homme.

DESCOLA, P., 1993 - Les lances du crépuscule. Relations Jivaros, Haute Amazonie, 506 p.; Paris: Plon. Collection Terre Humaine. 
HARNER, M. J., 1995 - Les Jivaros: Hommes des cascades sacrées, 198 p.; Paris: Petite Bibliothèque Payot 264.

ROSTAIN, S., 1999a - Excavación en área de un montículo de Huapula, proyecto Sangay-Upano. In: Memorias del Primer Congreso Ecuatoriano de Antropología, vol. 3: 227-256; Quito: Museo Jacinto Jijón y Caamaño/PUCE/MARKA.

ROSTAIN, S., 1999b - Secuencia arqueológica en montículos del valle del Upano en la Amazonía ecuatoriana. Bulletin de I'Institut Français d'Etudes Andines, 28 (1): 1-37; Lima.

ROSTAIN, S., 1999c - Occupations humaines et fonction domestique de monticules préhistoriques en haute Amazonie équatorienne. Bulletin de la Société Suisse des Américanistes, 63: 71-95; Neuchâtel.

ROSTAIN, S., 2000 - Analyse d'un sol d'habitat précolombien en Amazonie équatorienne. In: Peuplements anciens et actuels des forêts tropicales (Froment \& Guffroy, eds.): 142-146; Orléans : laboratoire ERMES-IRD.

TRUPP, F., 1981 - The Last Indians, 263 p.; Wörgl/Perlinger: South America's Cultural Heritage.

ZEIDLER, J. A., 1983 - La etnoarqueología de una vivienda Achuar y sus implicaciones arqueológicas. Boletín de los Museos del Banco Central del Ecuador, 3: 156-194; Quito: Miscelánea Antropológica Ecuatoriana. 